$16^{\text {th }}$ International Congress of Metrology, 11011 (2013)

DOI: $10.1051 /$ metrology/201311011

(C) Owned by the authors, published by EDP Sciences, 2013

\title{
Development of an Arduino shield for measurement and characterization of resistive sensors
}

\author{
Davide Aloisio $^{1}$, Nicola Donato ${ }^{1}$, Mariangela Latino ${ }^{1}$, Giovanni Neri ${ }^{1}$, Pier Paolo Capra ${ }^{2, a}$, Flavio Galliana ${ }^{2}$ \\ ${ }^{1}$ Dept. of Electronic Engineering and Industrial Chemistry and Engineering, University of Messina, Contrada di Dio, S. \\ Agata, 98166, Messina, Italy \\ 2 National Institute of Metrological Research, (INRIM), str. delle Cacce, 91, 10135 Turin, Italy
}

\begin{abstract}
In this paper is reported the activity regarding the development of a measurement system for Arduino DUE environment, able to perform electrical characterization of resistive sensing films under UV irradiation and to send data by USB and by Bluetooth protocols. The system is composed of two modular boards (Shields) the first for the electrical characterization, the latter for Bluetooth connection. The resistance variation of the sensor can be evaluated by properly choosing the capacitance value and by measuring the period (frequency) of a custom inverter-based oscillator. The GUI and the developed firmware are able to perform the real time monitoring of the sensor responses. The developed shield is able to measure the response of up to six sensors under UV radiation by means of LED devices. The GUIs were developed for the connections with Personal Computer, Tablet and Smartphone. The measurement apparatus was calibrated by measuring commercial resistors and then validated by characterizing Indium Oxides sensing films deposited by screen-printing technique on alumina substrates with platinum interdigitated electrodes on parallel configuration.
\end{abstract}

\section{Introduction}

Metal Oxides Based Sensors are probably the most promising devices working on gas monitoring, due to their qualities of low costs, easiness of use, sensing material deposition techniques and manufacturing technology compatible with Microelectronics and Micro Electro Mechanical Systems (MEMs) Industry. One of the most demanding requirements of such devices is the power required to set the operating temperature conditions, usually much higher than room temperature. Therefore, the goal is to develop devices working at temperature values close to room ones, this requirement can be fulfilled by choosing nanostructured sensing materials that can be photo-activated by UV irradiation $[1,2]$. In such scenario we develop a measurement system that can be used in the optimization of sensing devices under UV irradiation, in order to decrease the power required to achieve the right operating conditions. The system is low cost, open access, easy to upgrade and it can be used in both stand alone and in network configuration.

\section{Measurement system development}

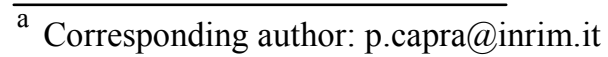

The sensor shield was developed by considering that a good choice to perform real time resistance measurements is to employ an oscillator circuit whose frequency is resistance value dependent [3]. This approach avoids use of A/D converters and simplifies the interfacing circuit of the sensor. The measurement system core is based on an oscillator circuit in which frequency (period) depends on the resistor value presents in the RC thank of the resonant ring. It's based on two inverter gate and a Schmidt trigger, essential for a proper RC charge and discharge, all connected in a loop for triple inversion of feedback signal. In this configuration, by choosing proper capacitance value, it is possible to adjust the range of measurement, see block diagram in Figure 1(a). The resistance measurement range spans from $1 \mathrm{k} \Omega$ to 100 $\mathrm{M} \Omega$, for the range shown in fig. 2 the reference capacitance value is $100 \mathrm{pF}$. The single sensor cell was then replicated to handle an array configuration by developing a sensor shield for Arduino DUE environment, fully compatible and able to be assembled with other ones in a modular structure. In Figure 1(b) is reported a picture of the system, the developed shields can be assembled in a stacked modular configuration.

Arduino DUE is a 32-bit open source board equipped with Atmel SAM3X8E ARM Cortex-M3 (84 MHz Clock), the hardware can handle both developed shields in a fast and accurate way. 
Figure 1. General Scheme (a) and picture (b) of the measurement System.

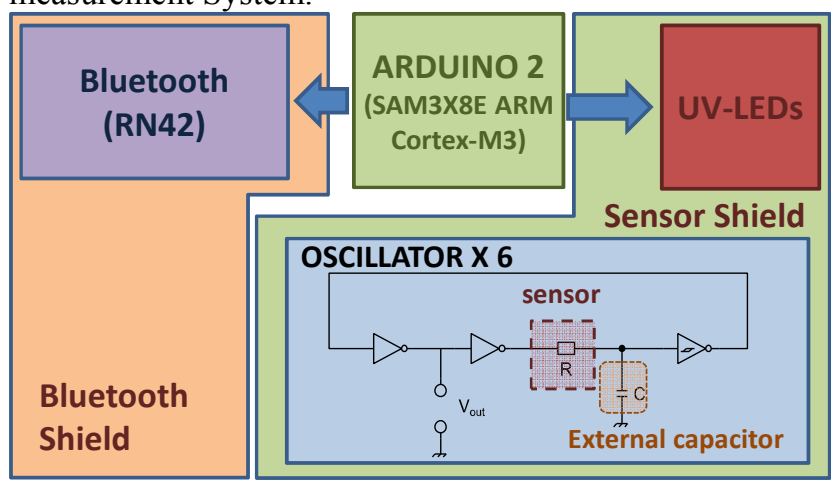

a)

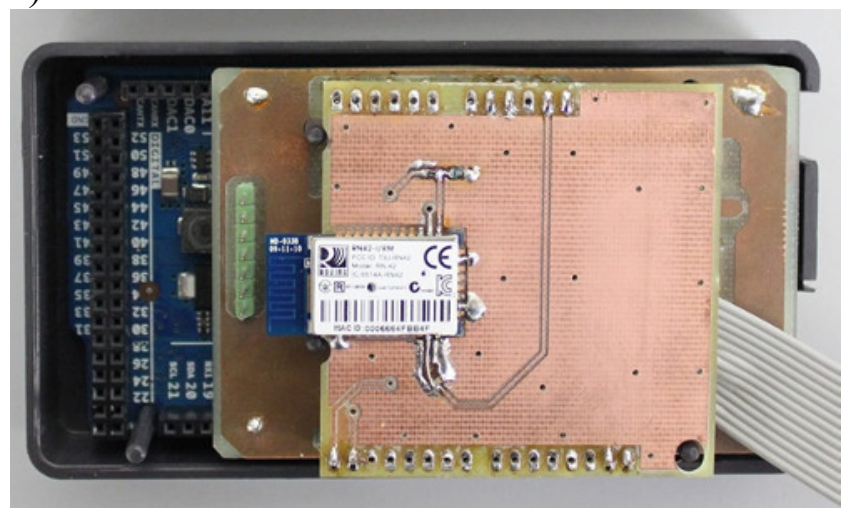

b)

Arduino DUE board, programmed with a proper developed library, provides both to read the frequency, by using internal interrupt events, and to send data by Bluetooth protocol. The Arduino firmware for frequency (period) measurement was validated by measuring signals coming from a SFG-830G Arbitrary Function Generator (from GWINSTEK).

The system was calibrated by measuring a set of precision commercial resistors, a look up table was written by taking into account the correspondence between the resistor value and the frequency (period) one, and then it was tested by measuring a new set of standard resistors measured with Agilent 34401A multimeter and with the measurement system. In fig. 2 it can be seen the comparison between the two data sets. The quality of the calibration procedure is assured by looking at the good agreement between the two data sets.

The Bluetooth Shield was developed by employing a Bluetooth Module (by Roving Networks), it allows the connection with a notebook by means of a GUI employed in Matlab environment (by MathWorks), and with tablets and smartphones equipped with Android operating system by means of a GUI developed in Java with Android Studio. Both the developed GUIs are able to control the communication protocol, the measurement procedures and the LEDs Switching.

\section{Results}

The samples are screen printed sensing films of Indium Oxide $\left(\operatorname{In}_{2} \mathrm{O}_{3}\right)$ deposited on alumina substrates equipped with interdigitated platinum electrodes on one side and with a platinum heater on the opposite side to set the operating temperature value ( Figure 3 ). Gas sensing tests were carried out inside a Teflon chamber equipped with $400 \mathrm{~nm}$ UV LEDs under controlled atmosphere. Mass flow controllers were used to adjust desired concentrations of target gas in dry air.

Figure 2. Comparison between the two data sets.

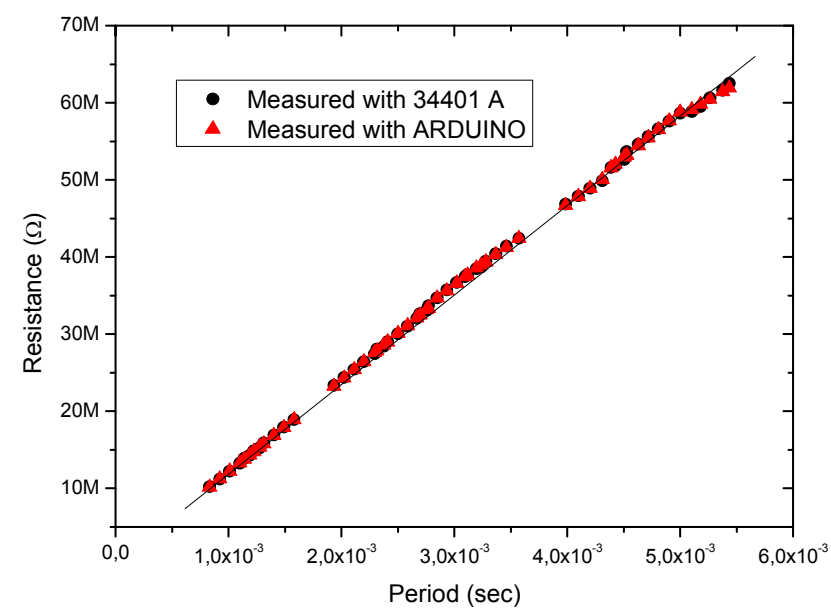

Figure 3. Sensor configuration: Top and Bottom layer.
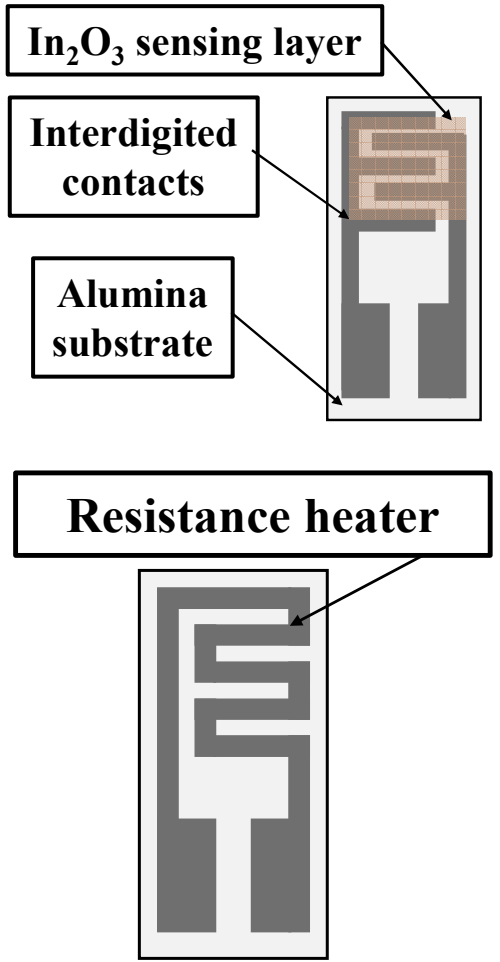

In Figure 4 can be seen the resistive behaviour of a $\operatorname{In}_{2} \mathrm{O}_{3}$ based sensor exposed in dry air atmosphere and dark/UV light conditions. It can be seen how in presence of UV irradiation the resistance suddenly decreases, then when the UV light is turned off (not reported), the resistance value slowly increases. 
Figure 4. Sample resistance under dry air and dark/radiation conditions.

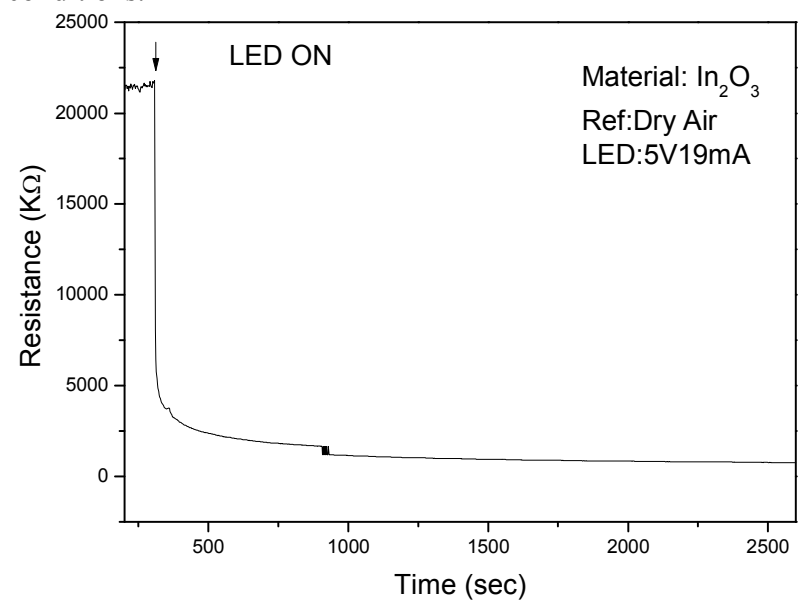

Figure 5. Resistance variation under $\mathrm{UV}$ radiation to a $\mathrm{NO}_{2}$ pulse (duration $250 \mathrm{~s}$ ) at room temperature (a) and at $100^{\circ} \mathrm{C}$ (b).
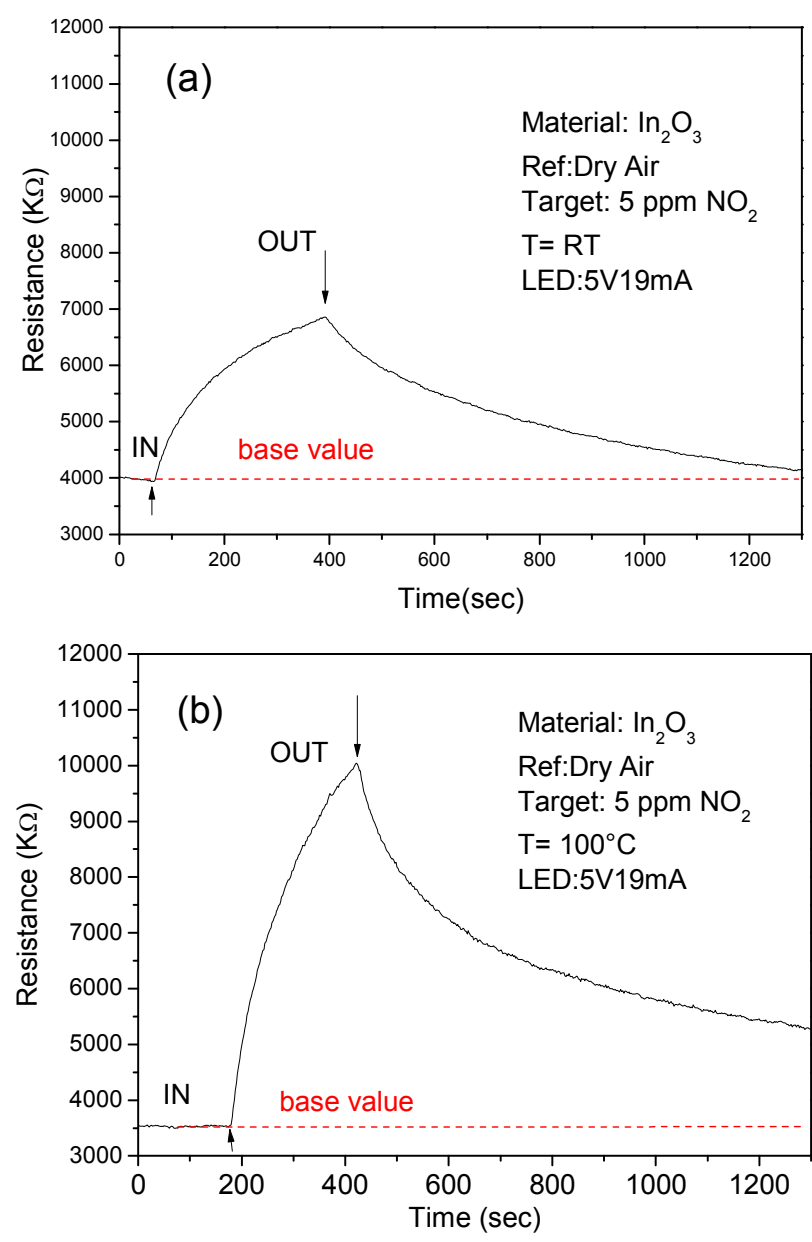

The sensors were tested with pulses of $5 \mathrm{ppm}$ of $\mathrm{NO}_{2}$ spanning from 10 to 250 seconds of duration. The biasing of the heater of the sensor is achieved with an Agilent 3631A power supply. In Figure 5 are reported the resistive behaviour of the same sample at room temperature (RT) and at $100^{\circ} \mathrm{C}$ (6.a and 6.b, respectively) when exposed to a 250 seconds pulse of 5 ppm of $\mathrm{NO}_{2}$ target gas. It can be seen how the higher temperature value brings to a lower base resistance (the resistance value measured in dry air conditions, $\mathrm{R}_{0}=3.5 \mathrm{M} \Omega$ ).
Figure 6. Response under UV radiation of sensing film vs. pulse duration of $5 \mathrm{ppm}$ of $\mathrm{NO}_{2}$ at RT.

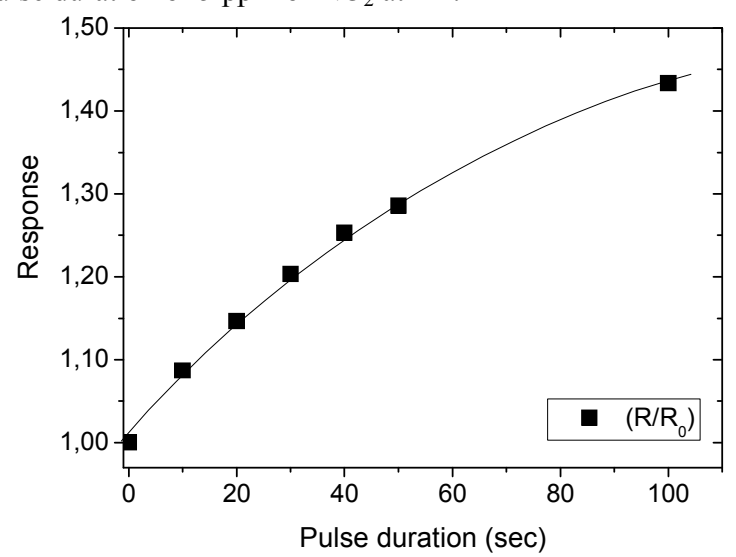

The sensing films acts as a n-type semiconductor, when exposed to oxidizing agent $\left(\mathrm{NO}_{2}\right.$ gas $)$ the resistance increases [4,5]. In Figure 6 is reported the response $\left(\mathrm{R} / \mathrm{R}_{0}\right)$ under UV radiation of sensing film vs. pulse duration of 5 ppm of $\mathrm{NO}_{2}\left(\mathrm{R}_{0}=4000 \mathrm{k} \Omega\right.$ in dry air $)$ at room temperature. The increasing of duration of the pulse brings to an increasing of the concentration of the gas adsorbed on film surface, with a consequent increasing of the response value.

\section{Conclusions}

Here is reported about the development of a measurement system for the characterization of resistive sensors with Arduino DUE environment. The developed shields are able to handle up to six sensors and six LEDs for UV irradiation and to send data by Bluetooth Protocol to notebooks, tablets and smartphones. The system was calibrated by measuring standard resistors, and then validated in the characterization of screen-printed $\mathrm{In}_{2} \mathrm{O}_{3}$ sensing films on alumina substrates towards $\mathrm{NO}_{2}$ as target gas. Further investigations are in progress in order to improve the shield by integrating a temperature monitor/control for the sensing films, allowing a deeper characterization of the operating conditions on several materials towards gas targets. The system is low cost, open access, easy to upgrade and it can be used in both stand alone and in network configuration.

\section{References}

1. T. Wagner, C.-D. Kohl, S. Morandi, C. Malagú, N. Donato, M. Latino, G. Neri, M. Tiemann, Chemistry - A European Journal,V. 18, Iss. 26, 25, pp. 8216-8223 (2012).

2. T. Wagner, C.-D. Kohl, C. Malagù, N. Donato, M. Latino, G. Neri, M. Tiemann, Sensors and Actuators B, (in press), http://dx.doi.org/10.1016/j.snb.2013.02.025

3. J. L. Merino, S. A. Bota, R. Casanova, A. Diéguez, C. Cané, J. Samitier, IEEE Trans. Instrum. Meas, vol. 53, no. 4, (2004).

4. George F. Fine, Leon M. Cavanagh, Ayo Afonja and Russell Binions, Sensors, 10, pp 5469-5502, doi:10.3390/s100605469, (2010).

5. N. Yamazoe and K. Shimanoe, Journal of Sensors, Vol. 2009), Article ID 875704, 21 pages doi:10.1155/2009/875704, (2009) 